\title{
Cientitifíca
}

http://revistas.udistrital.edu.co/ojs/index.php/revcie/index

\section{Retardo en Redes Inalámbricas Colaborativas}

\section{Delay in Collaborative Wireless Networks}

\section{Atraso em Redes Sem Fio Colaborativas}

\author{
César Augusto Hernández-Suárez ${ }^{1}$ \\ Diego Armando Giral-Ramírez ${ }^{2}$ \\ Luis Fernando Pedraza-Martínez ${ }^{3}$
}

Recibido: junio de 2019

Aceptado: noviembre de 2019

Para citar este artículo: Hernández-Suárez, C.A., Giral-Ramírez, D. A., Pedraza-Martínez, L.F. (2020). Retardo en Redes Inalámbricas Colaborativas. Revista Científica, 37(1), 122-130. Doi: https://doi.org/10.14483/23448350.15022

\section{Resumen}

La radio cognitiva es una tecnología que hace un uso eficiente del espectro y permite a los usuarios secundarios utilizar las oportunidades espectrales de las bandas licenciadas, pero sin interferir con las comunicaciones de los usuarios primarios. El objetivo de este artículo es analizar y comparar el desempeño de dos algoritmos de toma de decisiones multicriterio: Topsis y Vikor, con diferentes niveles de colaboración entre usuarios secundarios. Los dos algoritmos se evalúan y comparar con respecto al retardo durante una transmisión de información de 9 MB. La comparación se realizó bajo cuatro escenarios distintos, de acuerdo con la clase de servicio y el nivel de tráfico, para la banda de frecuencia GSM. De acuerdo con los resultados alcanzados el algoritmo Topsis tiene un mejor desempeño en los diferentes escenarios y niveles de colaboración.

Palabras clave: decisión espectral, GSM, MCDM, radio cognitiva, redes inalámbricas, retardo.

\begin{abstract}
Cognitive radio is a technology that makes efficient use of the spectrum allowing secondary users to use the spectral opportunities of the licensed bands, but without interfering with the communications of the primary users. The objective of this article is to analyze and compare the performance of two multicriteria decision making algorithms: Topsis and Vikor, with different levels of collaboration between secondary users. The two algorithms are evaluated and compared at the delay level during an information transmission of $9 \mathrm{MB}$. The comparison is made under four different scenarios, according to the class of service and the level of traffic, for the GSM frequency band. According to the results achieved, the Topsis algorithm has a better performance in different scenarios and collaboration levels.
\end{abstract} Keywords: cognitive radio, delay, GSM, MCDM, spectral decision, wireless networks.

Universidad Distrital Francisco José de Caldas. Bogotá, Colombia. cahernandezs@udistrital.edu.co Universidad Distrital Francisco José de Caldas. Bogotá, Colombia. dagiralr@correo.udistrital.edu.co Universidad Distrital Francisco José de Caldas. Bogotá, Colombia. Ifpedrazam@udistrital.edu.co 


\section{Resumo}

O rádio cognitivo é uma tecnologia que faz uso eficiente do espectro, permitindo que usuários secundários usem as oportunidades espectrais das bandas licenciadas, mas sem interferir nas comunicações dos usuários principais. O objetivo deste artigo é analisar e comparar o desempenho de dois algoritmos multicritério de tomada de decisão: Topsis e Vikor com diferentes níveis de colaboração entre usuários secundários. Os dois algoritmos são avaliados e comparados no nível de atraso durante uma transmissão de informação de $9 \mathrm{MB}$. A comparação é feita em quatro cenários diferentes, de acordo com a classe de serviço e o nível de tráfego, para a faixa de freqüência GSM. De acordo com os resultados obtidos, o algoritmo Topsis possui um melhor desempenho em diferentes cenários e níveis de colaboração.

Palavras-chaves: GSM, MCDM, decisão espectral, rádio Cognitivo, redes sem fio, atraso.

\section{Introducción}

En los últimos años las redes inalámbricas han sido de gran interés para la investigación debido al crecimiento de las tecnologías que utilizan el espectro para comunicarse. La demanda de usuarios y la evolución de las tecnologías han empezado a generar, de manera indirecta, escasez de bandas de frecuencias haciendo cada vez más compleja la asignación de espectro (López, García y Herrera, 2015).

El acceso dinámico al espectro es una tecnología que busca aprovechar aquellas bandas de frecuencia licenciadas que no sean utilizadas actualmente para hacer uso de ellas sin generar impactos en los usuarios licenciados (Akyildiz, Won-Yeol, Vuran y Mohanty, 2006). Lo anterior impone un desafío en el estudio de la utilización eficiente del espectro (Ahmed, Gani, Abolfazli, Yao y Khan, 2016; Akyildiz, Lee, Vuran y Mohanty, 2008; Akyildiz et al., 2006; Ozger y Akan, 2016; Tsiropoulos, Dobre, Ahmed y Baddour, 2016).

La radio cognitiva (CR) nace como una red de nueva generación que es capaz de cambiar sus parámetros de transmisión en función de su interacción para dar lugar a la negociación activa o comunicación con otros usuarios del espectro (Masonta, Mzyece y Ntlatlapa, 2013). Esta tecnología aumenta la capacidad de ancho de banda y el acceso dinámico al espectro garantizando que no existan interferencias entre usuarios primarios licenciados. (Akyildiz, Lee y Chowdhury, 2009; Tsiropoulos et al., 2016).

En la movilidad espectral el handoff espectral $(\mathrm{SH})$ se desarrolla a partir de las tres fases principales: medición, decisión y ejecución (Ahmed, Boulahia y Gaïti, 2014). En la fase de medición se realiza el descubrimiento de redes inalámbricas y la detección de oportunidades espectrales (SO) en dichas redes. Lo anterior se puede lograr a través de un enfoque centralizado o distribuido. En la fase de decisión, se toma la decisión del "cuándo" y "dónde" realizar el $\mathrm{SH}$, con base en los múltiples criterios y métricas que se hayan seleccionado. En la fase de ejecución se realiza la transferencia de la actual conexión a la nueva, teniendo en cuenta los requerimientos del handoff espectral mencionados anteriormente. De acuerdo con el modelo de handoff espectral que se configure, el desempeño de las redes de radio cognitiva $(\mathrm{CRN})$ puede ser afectado por factores como: latencia, throughput, señalización, interferencia, ancho de banda $(B W)$, relación señal a ruido más interferencia (SINR) y tasa de error de bit (BER), entre otros (Kumar, Prakash y Tripathi, 2016; Lertsinsrubtavee y Malouch, 2016).

Este artículo presenta una evaluación comparativa de dos algoritmos de toma de decisiones multicriterio: Topsis y Vikor en la banda de frecuencia GSM, para una red descentralizada. Los dos algoritmos se evalúan y comparan con respecto al retardo generado durante una transmisión de información de 9 MB. La comparación se realiza bajo cuatro escenarios distintos, de acuerdo con la clase de servicio (tiempo real y mejor esfuerzo) y el nivel de tráfico (alto y bajo): tiempo real (RT) con tráfico alto (HT), mejor esfuerzo (BE) con tráfico bajo (LT), RT con LT y BE con HT. El principal aporte del presente trabajo es considerar dentro del análisis 
diferentes niveles de colaboración (10\%, $20 \%$, $50 \%$, $80 \%$ y $100 \%$ ) entre usuarios secundarios, los cuales se comparten información espacio-temporal de la ocupación espectral que finalmente alimenta la base de datos de los algoritmos de toma de decisiones.

\section{Radio cognitiva}

De acuerdo con investigaciones previas (Federal Communications Commission, 2003), se ha comprobado que la mayor parte del espectro de radiofrecuencia se está utilizando de forma ineficaz. El análisis espacio-temporal evidencia que el uso del espectro asignado es ineficiente (Federal Communications Commission, 2003; IEEE Standards Coordinating Committee 41 on Dynamic Spectrum, 2008). Además, campañas de medición recientes revelan que más del $70 \%$ del espectro no está siendo utilizado (Hoven, Tandra y Sahai, 2005; Pedraza, Hernández, Galeano, Rodríguez-Colina y Páez, 2016).

Esta utilización ineficiente y esporádica del espectro, junto al incremento de la demanda del mismo, han causado que se degrade la calidad del servicio en varias redes y aplicaciones inalámbricas, como las comunicaciones móviles. Lo anterior ha motivado el desarrollo de investigaciones recientes, las cuales han encontrado en la asignación dinámica del espectro (DSA), la solución al problema. La tecnología clave que permite materializar las técnicas de DSA es la CR (Akyildiz et al., 2008; Tsiropoulos et al., 2016).

La CR tiene la capacidad de aprovechar de forma oportunista las porciones del espectro que no están siendo usadas en ese momento (Delgado y Rodríguez, 2016; Ozger y Akan, 2016). La figura 1 describe el concepto de SO a través de una gráfica en tres dimensiones, cuyos ejes son potencia, frecuencia y tiempo. Cada bloque gris de la figura es un PU que hace uso de una porción del espectro de frecuencia dado por el eje de frecuencia, a un nivel de potencia dado por el eje de potencia, y durante un determinado periodo dado por el eje de tiempo. Sin embargo, existen porciones del espectro de frecuencia que no son utilizadas durante determinado intervalo de tiempo, dichos espacios son denominados $\mathrm{SO}$, las cuales pueden ser aprovechadas por los SU (Ozger y Akan, 2016).

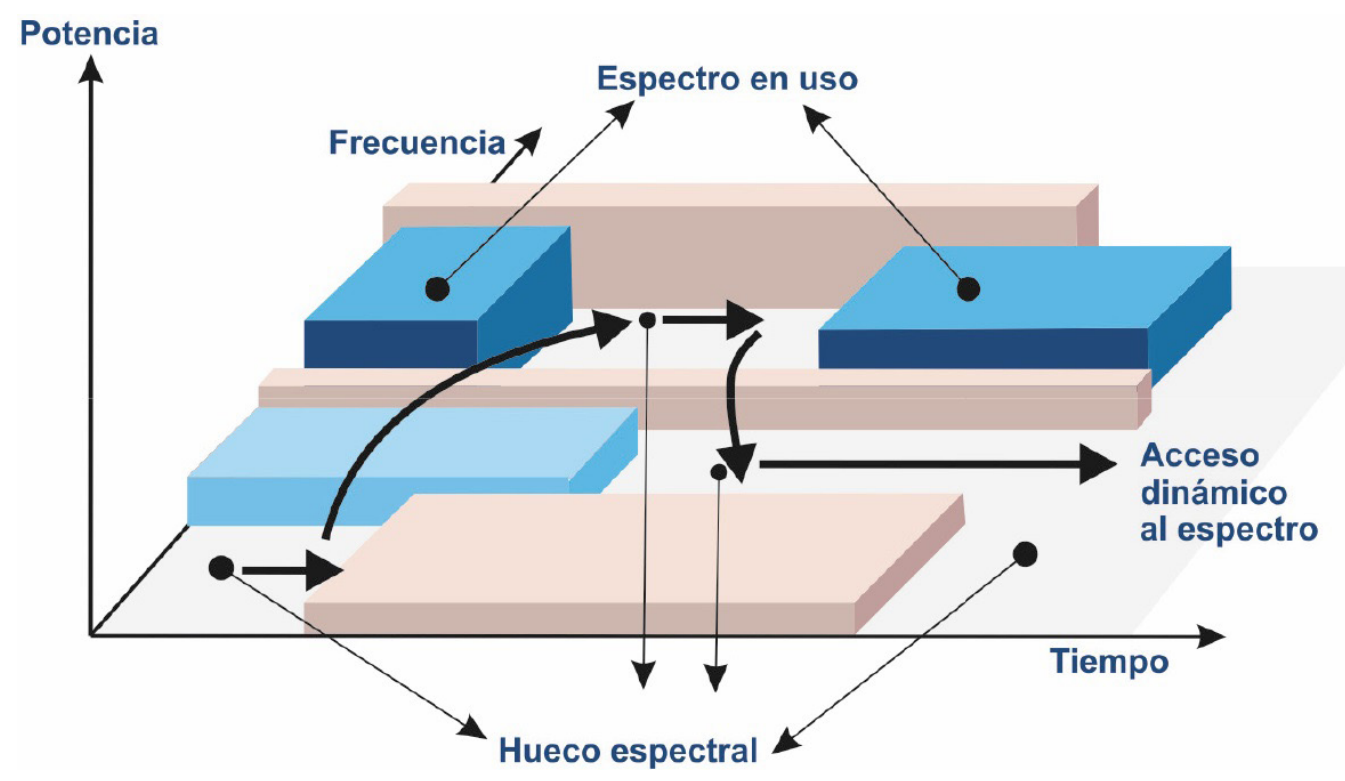

Figura 1. Concepto de SO.

Fuente: adaptada de Akyildiz et al. (2009). 


\section{Gestión de espectro en radio cognitiva}

La figura 2 describe el ciclo cognitivo, iniciando con la monitorización del espectro para detectar las $\mathrm{SO}$; luego, la decisión de espectro selecciona una de ellas para ser utilizada por el SU, si un PU arriba a la misma SO el SU debe realizar la movilidad espectral y seleccionar una nueva SO. En el caso de varios SU se puede realizar una compartición del espectro.

\section{Metodología}

Para determinar el desempeño de cada algoritmo se utilizó una herramienta de simulación desarrollada por los autores, en la cual el ambiente simulado reconstruye progresivamente el comportamiento de la ocupación espectral a partir de datos reales capturados en una campaña de medición previa en la banda de frecuencia GSM.

La información de ocupación espectral corresponde a una campaña de varias semanas de medición realizada en la ciudad de Bogotá, Colombia (Pedraza et al., 2016). La técnica de detección de energía se utilizó para determinar la matriz de disponibilidad de cada uno de los 124 canales de la banda GSM. El umbral de decisión para la potencia fue de $5 \mathrm{dBm}$ por encima del piso de ruido.

Si un SU desea realizar una transmisión durante $\phi$ minutos, la herramienta de simulación desarrollada, realiza el siguiente procedimiento:

- Primero: actualiza el valor de los DC con base en la información anterior al instante de tiempo (TS) actual, denominado $\tau$, en el cual el SU solicita el recurso espectral.

- Segundo: realiza un escalafón de clasificación de las SO con base en el puntaje obtenido por cada una, de acuerdo con la metodología del algoritmo de $\mathrm{SH}$ que se esté evaluando.

- Tercero: selecciona la SO que ocupe el primer lugar en el escalafón para asignársela al SU e iniciar su transmisión.

- Cuarto: en este momento, denominado $\tau 1$, se verifica en la base de datos (traza de datos capturados y procesados), si la SO seleccionada se encuentra disponible; si es así, se aumenta

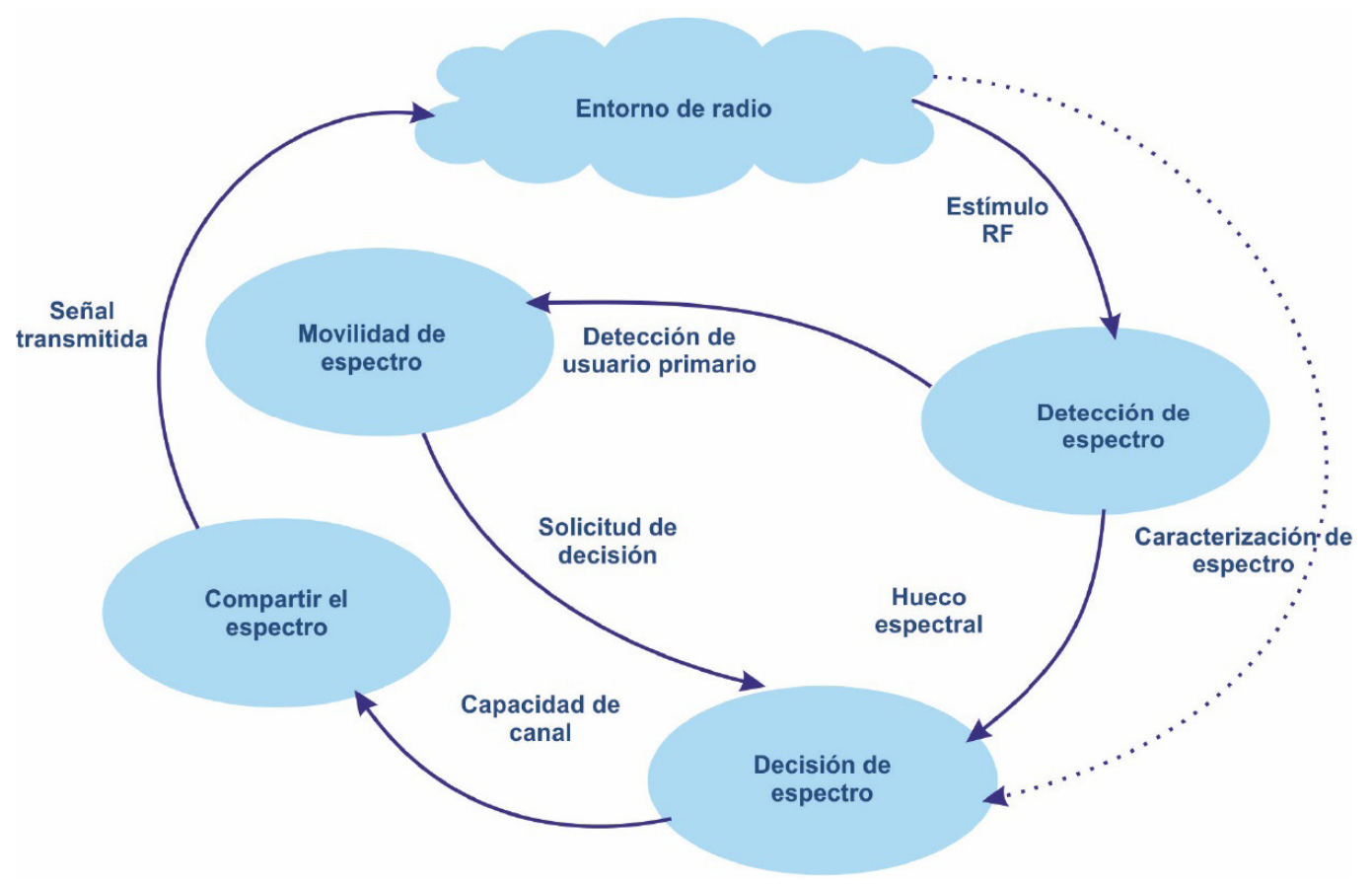

Figura 2. Ciclo cognitivo.

Fuente: adaptada de Akyildiz et al. (2009). 
en uno el número de handoffs y se procede al quinto paso, de lo contrario, se aumenta en uno el número de handoffs fallidos, se selecciona la SO que ocupe el siguiente lugar en el escalafón y se retorna al cuarto paso.

- Quinto: la herramienta de simulación verifica en cada TS, a través de la base de datos, si la SO que está siendo utilizada por el SU continua disponible.

- Sexto: en el momento $\tau$ k que la SO seleccionada sea requerida por un $\mathrm{PU}$, es decir, que la SO ya no se encuentra disponible según la base de datos, si $\Delta \tau=\tau \mathrm{k}-\tau 1$, es menor a 60 segundos, entonces se selecciona la siguiente $\mathrm{SO}$ en el ranking y se retorna al cuarto paso, en caso contrario, se actualiza $\tau 0$ con el valor de tiempo actual y se retorna al primer paso.

- Séptimo: si durante $\zeta$ segundos no se encuentra un canal disponible la comunicación se da por perdida.

La colaboración se definió para esta investigación como el intercambio de información entre SU con el objetivo de tener una mayor cantidad de información que permita realizar una mejor selección de la SO. La información a intercambiar se restringe a la ocupación espectral por parte del PU que el SU ha logrado detectar. Para el presente caso se asume una red descentralizada en la cual el nivel de colaboración entre SU juega un papel importante para el desempeño de la red.

\section{Algoritmos para asignación espectral}

Los dos algoritmos para toma de decisiones multicriterio (MCDM) seleccionados son: Technique for Order Preference by Similarity to Ideal Solution (Topsis) y Multi-Criteria Optimization and Compromise Solution (Vikor).

\section{Topsis}

Este algoritmo está basado en dos partes: la solución que no puede ser aceptada en ninguna situación y la solución ideal del sistema. Inicialmente, se construye la matriz de decisión $X$ y se normaliza usando el método de raíz cuadrada (ecuación 1) (Hernández, Giral, et al., 2015a; Ramírez y Ramos, 2013; Ramírez y Ramos, 2010).

$$
\tilde{X}=\left(\begin{array}{ccc}
\tilde{\chi}_{11} & \cdots & \tilde{\chi}_{1 M} \\
\vdots & \ddots & \vdots \\
\tilde{\chi}_{N 1} & \cdots & \tilde{\chi}_{N M}
\end{array}\right)=\left(\begin{array}{ccc}
\omega_{1} \tilde{\chi}_{11} & \ldots & \omega_{M} \tilde{\chi}_{1 M} \\
\vdots & \ddots & \vdots \\
\omega_{1} \tilde{\chi}_{N 1} & \cdots & \omega_{M} \tilde{\chi}_{N M}
\end{array}\right)
$$

Donde $\omega$ i es el peso asignado al criterio i, y la suma de estos debe ser 1 .

Luego, se determina la solución ideal y la peor solución, como lo describen las ecuaciones 2 y 3 .

$$
\begin{aligned}
& \mathrm{A}^{+}=\left\{\left(\max \tilde{\chi}_{\mathrm{ij}} \mid \mathrm{j} \in \mathrm{X}^{+}\right),\left(\min \tilde{\chi}_{\mathrm{ij}} \mathrm{j} \in \mathrm{X}^{-}\right)\right\}=\left\{\tilde{\chi}_{1}^{+}, \ldots, \tilde{\chi}_{\mathrm{M}}^{+}\right\} \\
& \mathrm{A}^{-}=\left\{\left(\min \tilde{\chi}_{\mathrm{ij}} \mathrm{j} \in \mathrm{X}^{+}\right),\left(\max \tilde{\chi}_{\mathrm{ij}} \mathrm{j} \in \mathrm{X}^{-}\right)\right\}=\left\{\tilde{\chi}_{1}^{-}, \ldots, \tilde{\chi}_{\mathrm{M}}^{-}\right\}
\end{aligned}
$$

Donde $\mathrm{i}=1, \ldots \mathrm{M}, \mathrm{y} X+\mathrm{y} X$ - son el conjunto de beneficios y costos, respectivamente.

Posteriormente, para cada alternativa se calcula la distancia euclidiana $\mathrm{D}$, como se observa en las ecuaciones 4 y 5 .

$$
\begin{array}{ll}
\mathrm{D}_{\mathrm{i}}^{+}=\sqrt{\sum_{\mathrm{j}=1}^{\mathrm{M}}\left(\tilde{\chi}_{\mathrm{ij}}-\tilde{\chi}_{\mathrm{j}}^{+}\right)^{2}} & \mathrm{i}=1, \ldots, \mathrm{N} \\
\mathrm{D}_{\mathrm{i}}^{-}=\sqrt{\sum_{\mathrm{j}=1}^{\mathrm{M}}\left(\tilde{\chi}_{\mathrm{ij}}-\tilde{\chi}_{\mathrm{j}}^{-}\right)^{2}} & \mathrm{i}=1, \ldots, \mathrm{N}
\end{array}
$$

Finalmente, las alternativas son organizadas en orden descendente, de acuerdo con el índice de preferencia dado por la ecuación 6.

$$
\mathrm{C}_{\mathrm{i}}^{+}=\frac{\mathrm{D}_{\mathrm{i}}^{-}}{\mathrm{D}_{\mathrm{i}}^{+}+\mathrm{D}_{\mathrm{i}}^{-}}, \quad \mathrm{i}=1, \ldots, \mathrm{N}
$$

\section{Vikor}

En palabras de Hernández, Páez y Giral: "El método VIKOR asume que cada alternativa es evaluada de acuerdo con cada función de criterio, y la clasificación puede ser desarrollada a través de la 
comparación de las medidas que estén más cercanas a la alternativa ideal" (2015, p. 33) .

En los trabajos de Hernández, Giral y Páez (2015b), Hernández, Páez et al. (2015); Ramírez y Ramos (2010), Stevens, Martinez y Pineda (2012) se describe el procedimiento del algoritmo VIKOR.

Para cada parámetro $\mathrm{j}=1,2,3, \ldots, \mathrm{N}$, se determina el mejor y peor valor, dados por las ecuaciones 7 y 8 .

$$
\begin{aligned}
& F_{j}^{+}=\left\{\left(\max _{i \in M} x_{i j} \mid j \in N_{b}\right),\left(\min _{i \in M} x_{i j} j \in N_{c}\right)\right\} \\
& F_{j}^{-}=\left\{\left(\min _{i \in M} x_{i j} j \in N_{b}\right),\left(\max _{i \in M} x_{i j} j \in N_{c}\right)\right\}
\end{aligned}
$$

Donde Nb y Nc pertenecen a N, y son los parámetros de beneficios y costos, respectivamente.

Luego, se calculan los valores de $R_{i}$ y $R_{i}$ para $\mathrm{i}=1$, $2,3, \ldots, M$, como lo describen las ecuaciones 9 y 10 .

$$
\begin{gathered}
S_{i}=\sum_{j \in N} w_{j} \frac{\left(F_{j}^{+}-x_{i j}\right)}{\left(F_{j}^{+}-F_{j}^{-}\right)} \\
R_{i}=\max _{i \in N}\left[w_{j} \frac{\left(F_{j}^{+}-x_{i j}\right)}{\left(F_{j}^{+}-F_{j}^{-}\right)}\right]
\end{gathered}
$$

Donde $Q_{i}$ es la importancia del peso del parámetro j.

Posteriormente, se calculan los valores de $Q_{i}$ para $i=1,2,3, \ldots, M$, dados por la ecuación 11 .

$$
\mathrm{Q}_{\mathrm{i}}=\gamma\left(\frac{\mathrm{S}_{\mathrm{i}}-\mathrm{S}^{+}}{\mathrm{S}^{-}-\mathrm{S}^{+}}\right)+(1-\gamma)\left(\frac{\mathrm{R}_{\mathrm{i}}-\mathrm{R}^{+}}{\mathrm{R}^{-}-\mathrm{R}^{+}}\right)
$$

Dónde, $\mathrm{S}^{+}=\min _{\mathrm{i} \in \mathrm{M}} \mathrm{S}_{\mathrm{i}}, \mathrm{S}^{-}=\max _{\mathrm{i} \in \mathrm{M}} \mathrm{S}_{\mathrm{i}}$, $\mathrm{R}^{+}=\min _{\mathrm{i} \in \mathrm{M}} \mathrm{R}_{\mathrm{i}}, \mathrm{R}^{-}=\max _{\mathrm{i} \in \mathrm{M}} \mathrm{R}_{\mathrm{i}}$, y $\quad 0 \leq \gamma \leq 1$.

Dados los valores de $\mathrm{Q}$ para todos los i pertenecientes a $\mathrm{M}$, las $\mathrm{SO}$ candidatas se clasifican de mayor a menor. Finalmente, la SO seleccionada está dada por el Q óptimo, como lo describe la ecuación 12.

$$
\mathrm{A}_{\mathrm{VIK}}^{*}=\arg \min _{\mathrm{i} \in \mathrm{M}} \mathrm{Q}_{\mathrm{i}}^{*}
$$

\section{Resultados}

En la evaluación de desempeño se tuvieron en cuenta dos clases de aplicaciones: tiempo real (RT) y mejor esfuerzo (BE); dos niveles de tráfico: tráfico alto (HT) y tráfico bajo (LT). Lo anterior, con la intención de formar cuatro tipos de escenarios: GSM RT HT, GSM RT LT, GSM BE HT y GSM BE LT; para la métrica de evaluación retardo promedio acumulado (AAD), tanto para el algoritmo Topsis (figura 3), como para el algoritmo Vikor (figura 4).

De la figura 3 se observa que, a excepción del escenario RT-HT, en todos se reduce el valor promedio del retardo conforme aumenta el nivel de colaboración: 11,24\% en el caso de BE-LT, 7,86 \% en el caso de RT-LT y 5,66 \% en el caso de BE-HT. De lo anterior se analiza que para tráfico bajo la mejoría en el desempeño del retardo es mayor que para tráfico alto, lo cual se explica por la menor cantidad de oportunidades espectrales. Otro hallazgo interesante es observar que el valor de retardo es muy similar entre enfoques BE y RT, para un mismo nivel de tráfico. En cuanto al porcentaje de colaboración entre usuarios secundarios se observa que su impacto está entre el $10 \%$ y el $12 \%$, de acuerdo con el escenario.

De la figura 4 se observa un comportamiento similar con el agravante que solo para el escenario BE-LT se observa un impacto positivo de la colaboración entre $\mathrm{SU}$, reduciendo el valor del retardo un $8,35 \%$ en el mejor caso.

\section{Conclusiones}

El nivel de colaboración no genera cambios significativos en los valores de desempeño del retardo para el algoritmo Vikor como en el caso de Topsis, donde el nivel de retardo se reduce entre un 10 y un $12 \%$, de acuerdo con el nivel de colaboración. Sin embargo, la tasa de cambio es relativamente baja.

En las CRN los SU deben tomar decisiones inteligentes en función de la variación del espectro y 

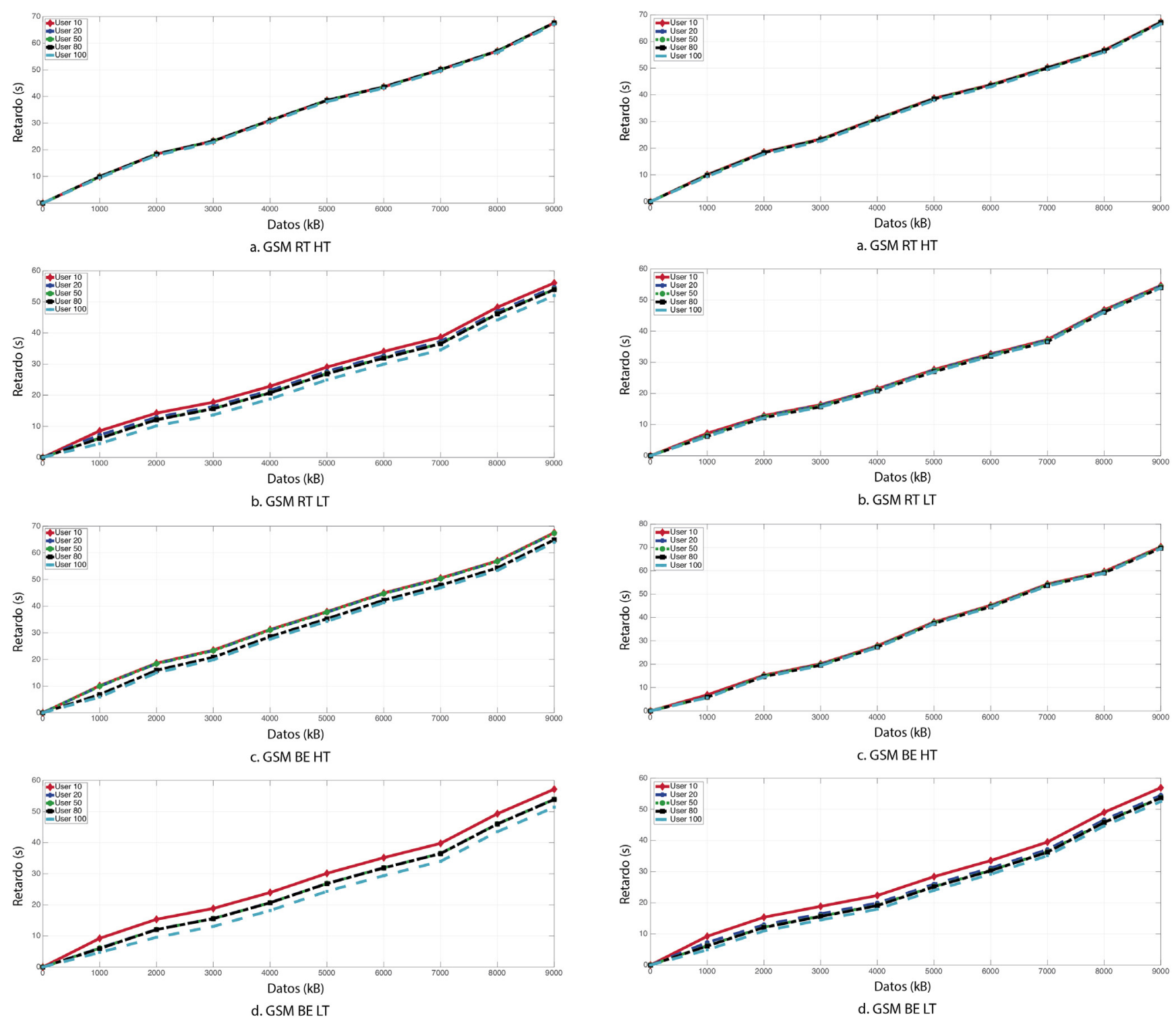

Figura 3. Retardo promedio acumulado para Topsis.

de las acciones adoptadas por otros SU. El desafío consiste en realizar el proceso de toma de decisiones para una DCRN dotando a los nodos con la capacidad de aprender del entorno, proponiendo estrategias que le permita a los SU intercambiar información de forma cooperativa o competitiva.

\section{Agradecimientos}

Los investigadores resaltan el soporte y financiamiento del proyecto de investigación por parte del

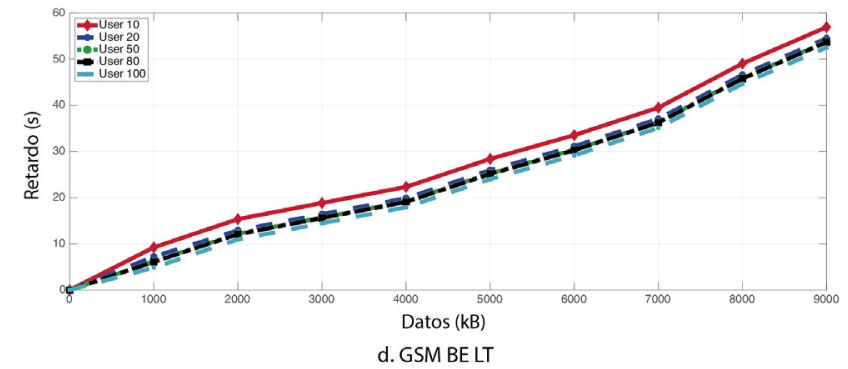

Figura 4. Retardo promedio acumulado para Vikor.

Centro de Investigaciones y Desarrollo Científico de la Universidad Distrital Francisco José de Caldas.

\section{Referencias}

Ahmed, A.; Boulahia, L. M.; Gaïti, D. (2014). Enabling vertical handover decisions in heterogeneous wireless networks: A state-of-the-art and a classification. IEEE Communications Surveys and Tutorials, 16(2), 776-811. http://doi. org/10.1109/SURV.2013.082713.00141 
Ahmed, E.; Gani, A.; Abolfazli, S.; Yao, L. J.; Khan, S. U. (2016). Channel assignment algorithms in cognitive radio networks: taxonomy, open issues, and challenges. IEEE Communications Surveys \& Tutorials, 18(1), 795-823. http://doi. org/10.1109/COMST.2014.2363082

Akyildiz, I. F.; Lee, W. Y.; Chowdhury, K. R. (2009). CRAHNs: Cognitive radio ad hoc networks. Ad Hoc Networks, 7(5), 810-836. http://doi. org/10.1016/j.adhoc.2009.01.001

Akyildiz, I. F.; Lee, W. -Y.; Vuran, M. C.; Mohanty, S. (2008). A survey on spectrum management in cognitive radio networks. IEEE Communications Magazine, 46(4), 40-48. http://doi. org/10.1109/MCOM.2008.4481339

Akyildiz, I. F.; Won-Yeol, L.; Vuran, M. C.; Mohanty, S. (2006). NeXt generation/dynamic spectrum access/cognitive radio wireless networks: A survey. Computer Networks, 50(13), 2127-2159. http://doi.org/10.1016/j.comnet.2006.05.001

Delgado, M.; Rodríguez, B. (2016). Opportunities for a more Efficient Use of the Spectrum based in Cognitive Radio. IEEE Latin America Transactions, 14(2), 610-616. http://doi.org/10.1109/ TLA.2016.7437200

Federal Communications Commission (2003). Notice of proposed rulemaking and order. Washington, D.C.: Federal Communications Commission.

Hernández, C.; Giral, D.; Páez, I. (2015a). Benchmarking of the Performance of Spectrum Mobility Models in Cognitive Radio Networks. International Journal of Applied Engineering Research (IJAER), 10(21).

Hernández, C.; Giral, D.; Páez, I. (2015b). Hybrid algorithm for frequency channel selection in wi-fi networks. World Academy of Science, Engineering and Technology, 9(12), 1212-1215.

Hernández, C.; Páez, I.; Giral, D. (2015). Modelo AHP-VIKOR para handoff espectral en redes de radio cognitiva. Tecnura, 19(45), 29-39.

Hernández, C.; Vásquez, H.; Páez, I. (2015). Proactive spectrum handoff model with time series prediction. International Journal of Applied Engineering Research (IJAER), 10(21), 42259-42264.

Hoven, N.; Tandra, R.; Sahai, A. (2005). Some fundamental limits on cognitive radio. Wireless Foundations EECS. Berkeley: University of California.

IEEE Standards Coordinating Committee 41 on Dynamic Spectrum (2008). 1900.1-2008 - IEEE standard definitions and concepts for dynamic spectrum access: terminology relating to emerging wireless networks, system functionality, and spectrum management.

IEEE Standard 1900.1-2008. https://standards.globalspec.com/std/13285031/1900.1

Kumar, K.; Prakash, A.; Tripathi, R. (2016). Spectrum handoff in cognitive radio networks: a classification and comprehensive survey. Journal of Network and Computer Applications, 61, 161-188. http://doi.org/10.1016/j. jnca.2015.10.008

Lertsinsrubtavee, A.; Malouch, N. (2016). Hybrid spectrum sharing through adaptive spectrum handoff and selection. IEEE Transactions on Mobile Computing. http://doi.org/10.1109/ $\underline{\text { TMC.2016.2517619 }}$

López, D. A.; García, N. Y.; Herrera, J. F. (2015). Desarrollo de un modelo predictivo para la estimación del comportamiento de variables en una infraestructura de Redred. Información Tecnológica, 26(5), 143-154.

Masonta, M. T.; Mzyece, M.; Ntlatlapa, N. (2013). Spectrum decision in cognitive radio networks: a survey. IEEE Communications Surveys \& Tutorials, 15(3), 1088-1107. http://doi.org-/10.1109/ $\underline{\text { SURV.2012.111412.00160 }}$

Ozger, M.; Akan, O. B. (2016). On the utilization of spectrum opportunity in cognitive radio networks. IEEE Communications Letters, 20(1), 157-160. http://doi.org/10.1109/ LCOMM.2015.2504103

Pedraza, L. F.; Hernández, C.; Galeano, K.; Rodríguez-Colina, E.; Páez, I. P. (2016). Ocupación 
espectral y modelo de radio cognitiva para Bogotá. Bogotá: Editorial UD.

Ramírez, C.; Ramos-R., V. (2013). On the effectiveness of multi-criteria decision mechanisms for vertical handoff. En International Conference on Advanced Information Networking and Applications (pp. 1157-1164). http://doi. org/10.1109/AINA.2013.114

Ramírez, C.; Ramos, V. M. (2010). Handover vertical: un problema de toma de decisión múltiple. En Congreso Internacional sobre Innovación y Desarrollo Tecnológico.
Stevens-Navarro, E.; Martínez-Morales, J. D.; Pineda-Rico, U. (2012). Evaluation of vertical handoff decision algorightms based on MADM methods for heterogeneous wireless networks. Journal of Applied Research and Technology, 10(4), 534-548.

Tsiropoulos, G.; Dobre, O.; Ahmed, M.; Baddour, K. (2016). Radio Resource Allocation Techniques for Efficient Spectrum Access in Cognitive Radio Networks. IEEE Communications Surveys \& Tutorials, 18(1), 824-847. http://doi. org/10.1109/COMST.2014.2362796 